City University of New York (CUNY)

CUNY Academic Works

2018

\title{
Analysis of Episodes of Care in Medicare Beneficiaries Newly Diagnosed with Alzheimer's Disease
}

Rezaul K. Khandker

Merck \& Co.

Christopher Black

Merck \& Co

Lin Xie

STATinMED Research

M. Furaha Kariburyo

STATInMED Research

Baishali M. Ambegaonkar

Merck \& Co.

See next page for additional authors

\section{How does access to this work benefit you? Let us know!}

More information about this work at: https://academicworks.cuny.edu/ny_pubs/454

Discover additional works at: https://academicworks.cuny.edu

This work is made publicly available by the City University of New York (CUNY).

Contact: AcademicWorks@cuny.edu 


\section{Authors}

Rezaul K. Khandker, Christopher Black, Lin Xie, M. Furaha Kariburyo, Baishali M. Ambegaonkar, Onur

Baser, Huseyin Yuce, and Howard Fillit 


\title{
Analysis of Episodes of Care in Medicare Beneficiaries Newly Diagnosed with Alzheimer's Disease
}

\author{
Rezaul K. Khandker, PhD, "Christopher M. Black, MPH, " Lin Xie, MS, ${ }^{\dagger}$ \\ M. Furaha Kariburyo, MPH, Baishali M. Ambegaonkar, PhD, * Onur Baser, PhD, ${ }^{\dagger}$ \\ Huseyin Yuce, PhD, ${ }^{\S}$ and Howard Fillit, $M D^{\text {II/I }}$
}

OBJECTIVES: To study transitions between healthcare settings and quantify the cost burdens associated with different combinations of transitions during a 6-month period before initial Alzheimer's disease (AD) diagnosis so as to investigate how using an episode-of-care approach to payment for specific disease states might apply in AD.

DESIGN: A retrospective observational cohort study.

SETTING: United States.

PARTICIPANTS: A random sample of 8,995 individuals aged 65 to 100 with a diagnosis of AD (International Classification of Diseases, Ninth Revision, Clinical Modification code 331.0) were identified from the Medicare database between January 1, 2011, and June 30, 2014. This analysis identified individuals with $\mathrm{AD}$ diagnosed in inpatient $(18 \%)$, skilled nursing facility (SNF) $(1 \%)$, hospice $(4 \%)$, and home and outpatient $(77 \%)$ settings and analyzed episodes that began in the index setting (defined as the care setting in which the individual was first diagnosed with AD).

MEASUREMENTS: Study outcomes included number of transitions between settings, primary discharge diagnoses, and total all-cause healthcare costs during the 6 months after the AD diagnosis.

RESULTS: The average numbers of transitions between care settings were 2.8 originating from an inpatient setting, 2.4 from a SNF, 0.3 from a hospice setting and 0.7 from a home or outpatient setting during 6 months postAD diagnosis. The overall cost burden during the 6 months after $\mathrm{AD}$ diagnosis (including costs incurred at the index setting) was high for individuals diagnosed in a

From the *Merck \& Co., Inc., Kenilworth, New Jersey, USA;

${ }^{\dagger}$ TATinMED Research, Ann Arbor, Michigan; ${ }^{\ddagger}$ Center for Innovation and Outcomes Research, Department of Surgery, Columbia University;

$\S_{\text {New }}$ York City College of Technology, City University of New York;

"I Icahn School of Medicine at Mount Sinai; and the "Alzheimer's Drug

Discovery Foundation, New York, New York.

Study was funded by Merck \& Co., Inc., Kenilworth, NJ USA.

Address correspondence to Rezaul Khandker, PhD, MBA, Merck \& Co., Inc., 2000 Galloping Hill Rd, Kenilworth, NJ 07033 e-mail: rezaul.

khandker@Merck.com

DOI: 10.1111 /jgs.15281 nonambulatory setting (mean $\$ 41,468$ ). Individuals diagnosed in an ambulatory setting incurred only $\$ 12,597$ in costs during the same period.

CONCLUSION: Episodes of care can be defined and studied in individuals with AD. An episode-of-care approach to payment could encourage providers to use the continuum of care needed for quality medical management in AD more efficiently. J Am Geriatr Soc 66:864-870, 2018.

Key words: Alzheimer's disease; health economics; episodes of care

$I^{n}$ $\mathrm{n}$ the current U.S. healthcare environment, policymakers are searching for alternative payment models to improve quality of care, promote efficiency, and reduce healthcare spending. ${ }^{1,2}$ Episode-based payment, also known as bundled payments, are payment models designed to pay a single price for all services that a person needs during an entire episode of care. The goal is to provide incentives to healthcare providers to reduce unnecessary services within each episode of care by providing better care at lower costs. ${ }^{3}$ To our knowledge, no studies have evaluated episode-based payment methods associated with Alzheimer's disease (AD). An estimated 5.4 million individuals in the United States are currently diagnosed with $\mathrm{AD}$, with approximately 5 million of them being aged 65 and older. ${ }^{4}$ More than 15 million family members and other unpaid caregivers provided an estimated 18 billion hours of care to people with $\mathrm{AD}$ and other dementias, a contribution valued at more than \$217 billion in 2014 . In 2016, total healthcare costs, including long-term care and hospice, for people with dementia were estimated to be approximately $\$ 236$ billion, with a steady increase from 2013 to $2016 .^{5-8}$ One way to understand and analyze the longitudinal healthcare cost of $\mathrm{AD}$ is to categorize these costs into distinct episodes of care. An episode of care can be defined as a series of temporally contiguous 
healthcare services related to the treatment of a disease through interactions between individuals with the disease and providers. ${ }^{9,10}$

This study analyzed episodes of care for Medicare beneficiaries newly diagnosed with $\mathrm{AD}$. The purpose was to describe transitions between healthcare settings and to examine and quantify the cost associated with different combinations of transitions during the 6 months after initial AD diagnosis. An objective of this study was to explore, using an episode-of-care approach, whether novel methods of payment for dementia care might be possible as a way to promote better quality of care.

\section{METHODS}

\section{Data Source}

A retrospective analysis was performed of individuals newly diagnosed with $\mathrm{AD}$ using the Medicare 5\% national sample administrative database from January 2010 through December 2014. Medicare claims data files used in this study included inpatient and outpatient, Medicare carrier, Part D drug events, skilled nursing facility (SNF), home health agency (HHA), hospice, durable medical equipment (DME), and Medicare denominator file, which contains demographic and enrollment information of Medicare beneficiaries.

\section{Participant Identification}

Beneficiaries aged 65 to 100 and diagnosed with 1 primary or 2 or more secondary diagnoses for AD (International Classification of Diseases, Ninth Edition, Clinical Modification (ICD-9-CM) code 331.0) during the identification period (January 1，2011-June 30, 2014) were included in the study. The first AD diagnosis date was designated as the index date. Participants were also required to have continuous health plan enrollment with medical and pharmacy benefits for at least 12 months before (baseline period) and 6 months after (follow-up period) the index date. Individuals with pharmacy claims for an antidementia drug (donepezil, rivastigmine, galantamine, memantine) or claims of $\mathrm{AD}$ or $\mathrm{AD}$-related dementia (ICD-9-CM codes 290.xx, 294.xx, 331.1, 331.2, 331.7, $331.82,331.89,331.9,797)$ during the baseline period were excluded. This analysis identified individuals diagnosed in inpatient, SNF, hospice, and home or outpatient settings. Home and outpatient settings included outpatient, DME, HHA, and Medicare Carrier outpatient settings.

\section{Episode-of-Care Measurement}

This retrospective study was two faceted. The first facet was to examine transitions between settings and overall cost burden during the 6 months after $\mathrm{AD}$ diagnosis in individuals newly diagnosed with $\mathrm{AD}$ in inpatient, $\mathrm{SNF}$, hospice, and home and outpatient settings. The second facet was to examine and quantify cost burden associated with common combinations and to evaluate admitting diagnoses. We analyzed episodes that began in the setting where $\mathrm{AD}$ was first diagnosed and then studied participant pathways and transitions of care between settings for up to 3 transitions during the 6 months after the $\mathrm{AD}$ diagnosis. All transitions between settings for each participant were then collated into one "combination". This analysis focused on the 4 most commonly observed combinations in our study sample.

\section{Outcomes Variables}

Study outcomes included the number of transitions between settings, total all-cause healthcare costs, and costs for the first 3 transitions during the 6 months after AD diagnosis. Total costs were censored at 6 months. For the 4 most frequent combinations of transition, we analyzed length of stay (LOS), primary diagnoses, and healthcare costs of the first 3 settings in the combination. The discharge diagnoses coded on inpatient, SNF, and hospice claims were captured. The principal diagnosis coded on an outpatient claim was used as the primary diagnosis in an outpatient setting.

\section{Baseline Variables}

Baseline characteristics from 12 months before an $\mathrm{AD}$ diagnosis (age, race, sex, U.S. geographical region, individual comorbid conditions) were evaluated.

\section{Statistical Analysis}

All study variables were examined descriptively. Numbers and percentages were determined for dichotomous and polychotomous variables. Means and standard deviations were determined for continuous variables.

\section{RESULTS}

\section{Participant Characteristics}

After applying the study selection criteria, 8,995 individuals newly diagnosed with AD aged 65 to 100 were selected for analysis: $18 \%$ in inpatient settings, $77 \%$ in home and outpatient settings, $1 \%$ in SNFs, and $4 \%$ in hospice (Supplementary Figure S1).

The overall mean age was $84,82 \%$ of participants were white, $77 \%$ were female, $37 \%$ resided in the South at the time of diagnosis. The most common comorbidities were hypertension $(55 \%)$, diabetes $(26 \%)$, chronic pulmonary disease $(19 \%)$, cerebrovascular disease $(18 \%)$, and mood disorder (16\%) (Supplementary Table S1).

\section{Transitions of Care Between Settings}

\section{Inpatient}

During the 6 months after AD diagnosis, the average number of transitions was 2.8 for 1,637 individuals with $\mathrm{AD}$ diagnosed in the inpatient setting $(18 \%)$. Approximately half of the individuals diagnosed in inpatient settings transitioned to a SNF and $49 \%$ to home and outpatient settings. Of those who transitioned to a SNF, approximately $14 \%$ were readmitted to the hospital, and $78 \%$ were 


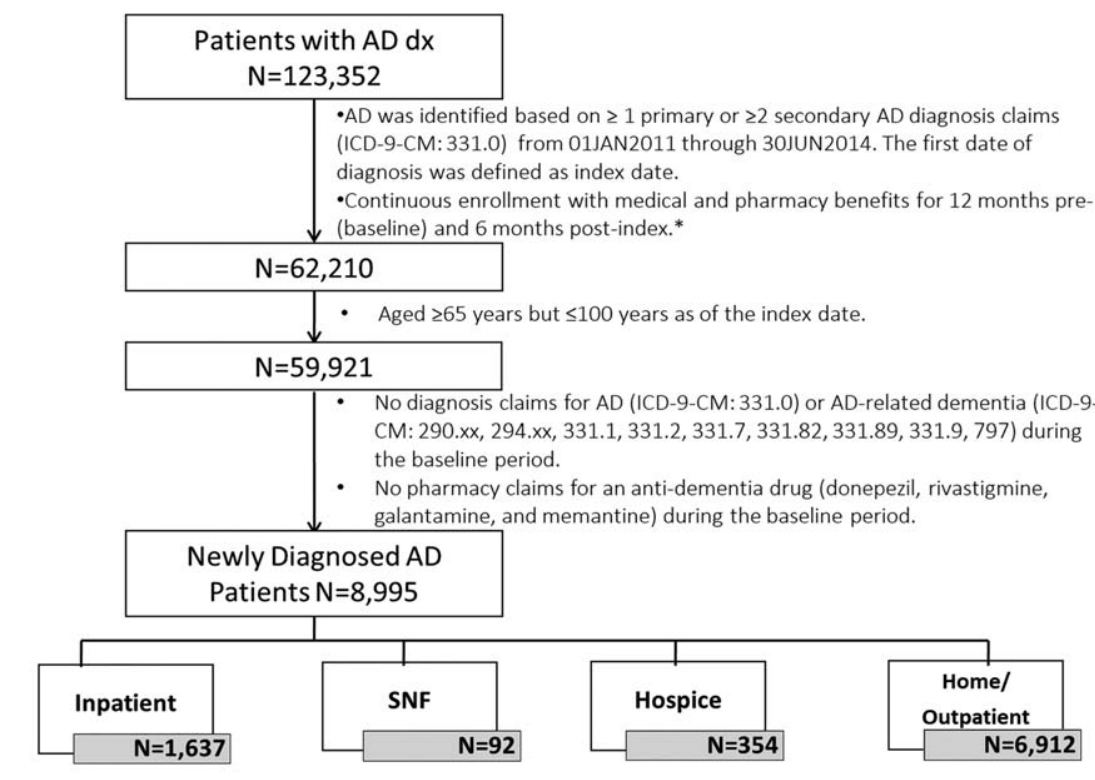

*Patient data was assessed until the earliest of death, health plan disenrollment, or 31DEC2014

Figure 1. Flow chart for participant selection criteria.

discharged home. Of those who were discharged to home or an outpatient setting, $58 \%$ did not have a second transition, and almost 37\% were re-hospitalized. The 30-day hospital readmission rate after index hospitalization discharge was $22 \%$. Participants who remained in the home or outpatient setting and did not have a second transition had almost \$30,000 lower costs than those who were readmitted to the hospital (Figure 2a).

\section{Home and outpatient}

Most individuals newly diagnosed with AD were diagnosed in the home or outpatient setting $(n=6,912)$ and had an average of 0.7 transitions between care settings during the 6 months after $\mathrm{AD}$ diagnosis. Of participants diagnosed in home and outpatient settings, $76.5 \%$ remained in the original setting and did not transition to another care setting, and $19.6 \%$ were later admitted to the hospital during the 6 months after AD diagnosis. The cost of care was $\$ 46,941$ more for participants who had a second transition from inpatient to SNF than for those who remained in the home or outpatient setting and $\$ 24,308$ more for those who had a second transition from inpatient to home or outpatient (Figure 2b).

\section{Skilled Nursing Facility}

Participants who were initially diagnosed in a SNF $(n=92)$ had an average of 2.4 transitions between care settings over the 6 months after AD diagnosis. Seventy-seven percent of participants diagnosed in a SNF were discharged to a home or outpatient setting, and $18.5 \%$ were admitted to the hospital (Figure 3a).

\section{Hospice}

The mean number of transitions between care settings was 0.3 for participants originally diagnosed with $\mathrm{AD}$ in a hospice setting $(n=354)$. More than three-quarters of these participants remained in hospice and did not transition to another setting during the 6 months after $\mathrm{AD}$ diagnosis. Total Medicare expenditures were \$30,169 for those who did not transition to another setting during the 6 months after $\mathrm{AD}$ diagnosis and $\$ 46,223$ for those who had their first transition to an inpatient setting and then to a SNF (Figure 3b).

\section{Primary Discharge Diagnoses Associated with Commonly Observed Combinations of Transitions}

Four commonly observed combinations of transitions were further evaluated.

\section{Home or Outpatient Setting $\rightarrow$ Inpatient Setting $\rightarrow$ Home or Outpatient Setting}

The first combinations included 765 participants who transitioned from home or outpatient to inpatient and back to home or outpatient setting. The primary diagnoses were $\mathrm{AD}$ for index home or outpatient, malaise or fatigue for inpatient setting, and diabetes for second home or outpatient settings (Supplementary Figure S1).

Inpatient Setting $\rightarrow$ SNF Setting $\rightarrow$ Home or Outpatient Setting

The second combination included 646 participants who went from inpatient to SNF then home or outpatient setting. The first primary discharge diagnosis in the index inpatient setting was malaise or fatigue. $\mathrm{AD}$ was not recorded in the top 9 primary discharge diagnoses in the inpatient setting. AD was the principal discharge diagnosis in the SNF setting, and hypertension was the primary diagnosis for participants in the home or outpatient setting (Supplementary Figure S2). 
A.

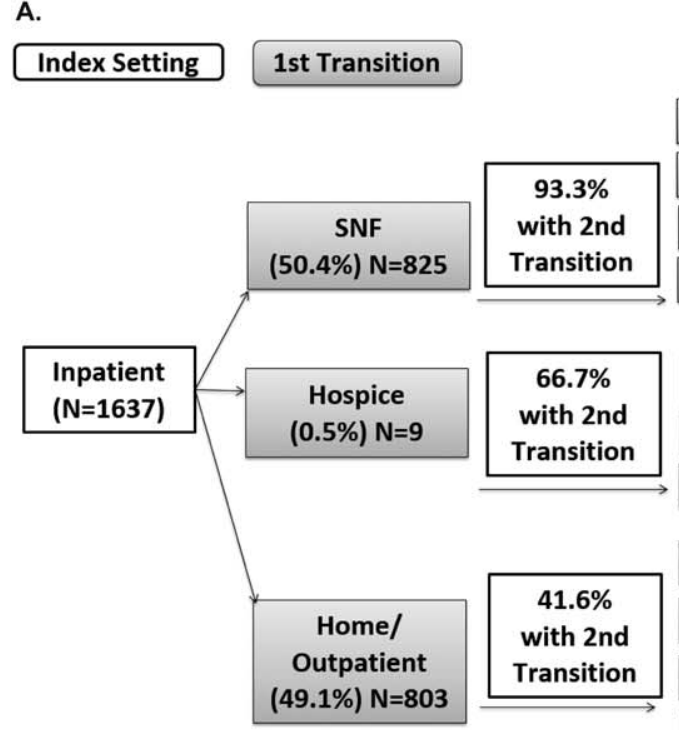

B.

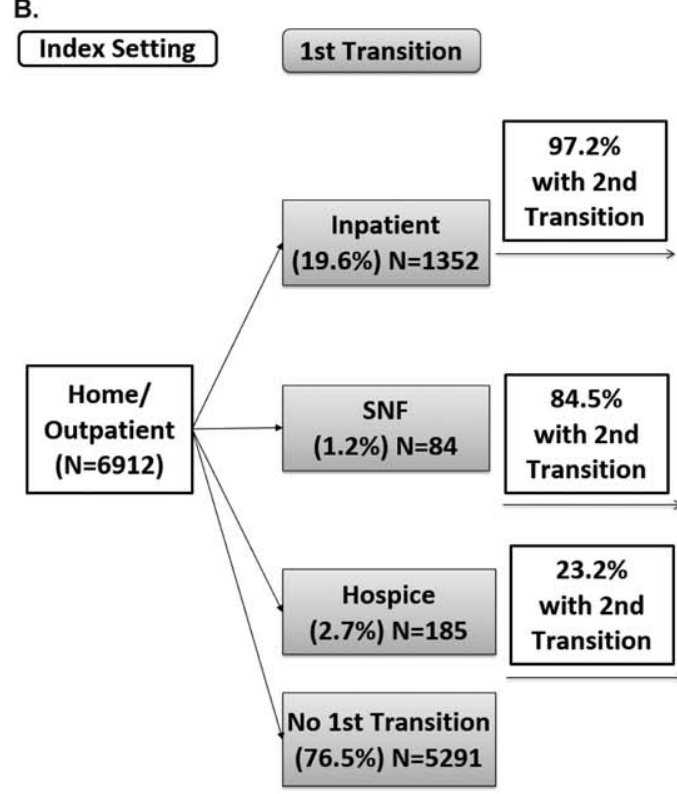

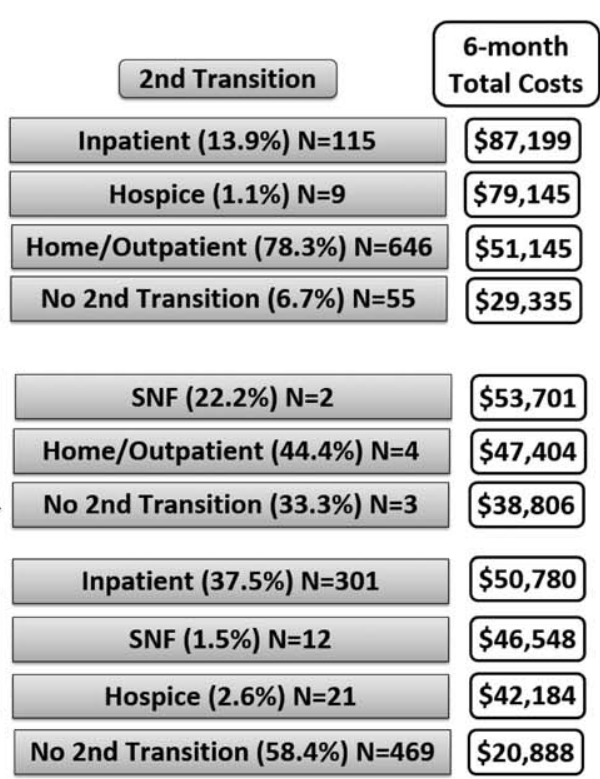

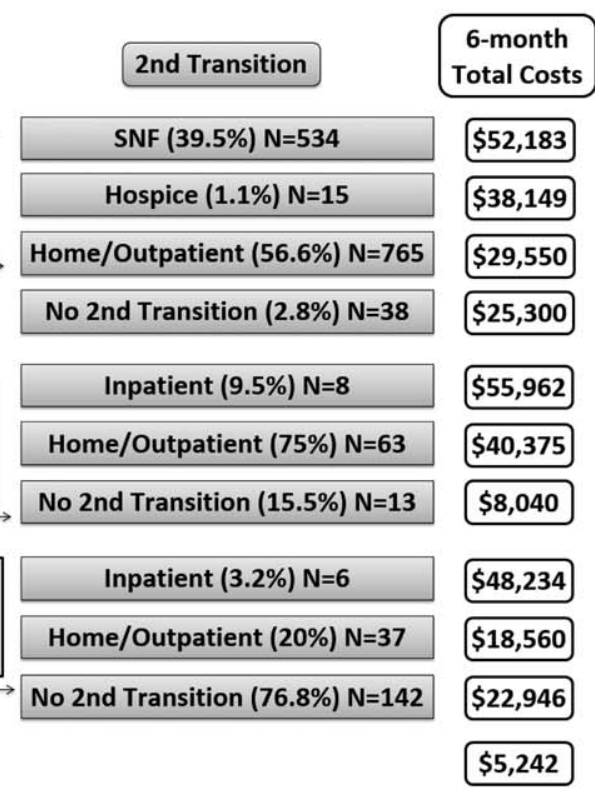

Figure 2. Transition from (A) inpatient, (B) home or outpatient.

Home or Outpatient Setting $\rightarrow$ Inpatient Setting $\rightarrow$ SNF Setting

Participants who transitioned from home or outpatient to inpatient and then SNF setting were captured in the third most commonly observed combination of transitions and included 534 individuals with AD. The primary diagnoses were $\mathrm{AD}$ in the index home or outpatient setting, pneumonia in the inpatient setting, and hypertension in the SNF (Supplementary Figure S3).

\section{Inpatient Setting $\rightarrow$ Home or Outpatient Setting $\rightarrow$ Inpatient Setting}

The fourth combination included 301 participants who transitioned from an inpatient to a home or outpatient and back to an inpatient setting. The primary discharge diagnosis in the index inpatient setting was syncope and collapse, diabetes mellitus in the home or outpatient setting, and chest pain in the second inpatient setting (Supplementary Figure S4).

\section{Cost Associated with Commonly Observed} Combinations of Transitions

Home or Outpatient Setting $\rightarrow$ Inpatient Setting $\rightarrow$ Homel Outpatient Setting

The average total Medicare expenditures of the first combination was $\$ 29,550$ over 6 months. The average LOS was 74 days in the first home or outpatient setting, which accounted for $14.6 \%(\$ 4,322)$ of the average total cost. The next transition was to an inpatient setting, where participants remained an average of 6 days, accounting for $31.3 \%(\$ 9,250)$ of the total cost. The average length of 
A.

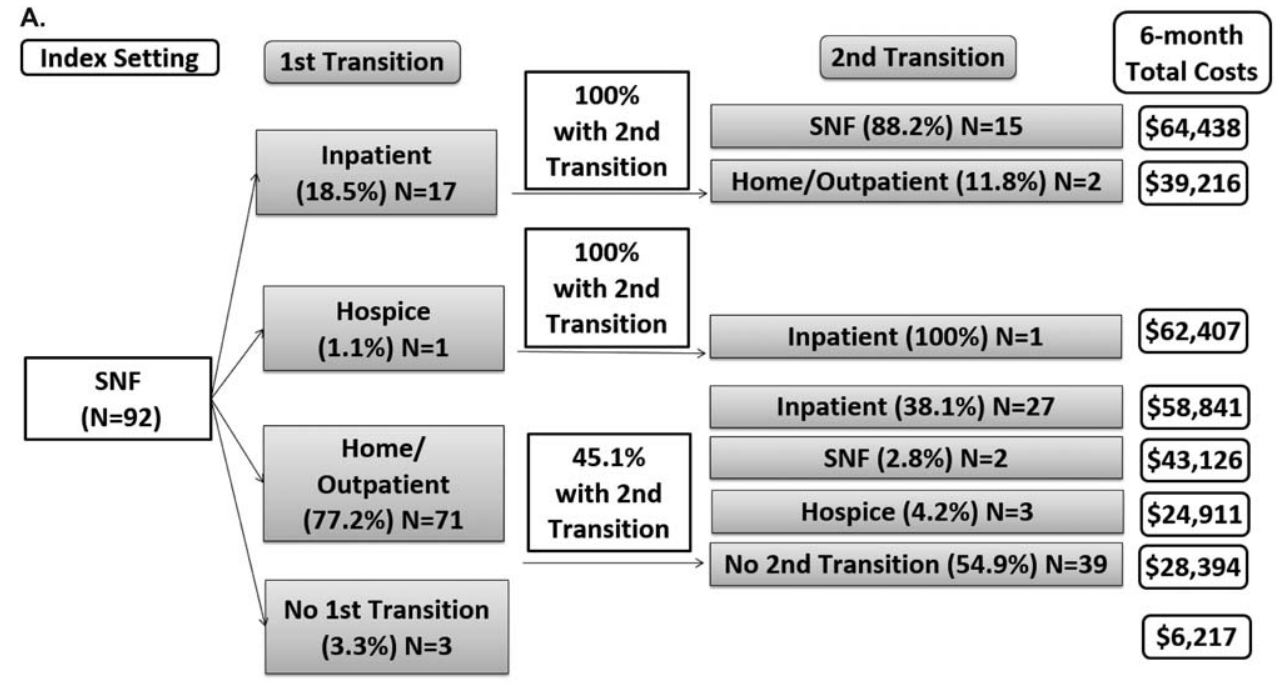

B.

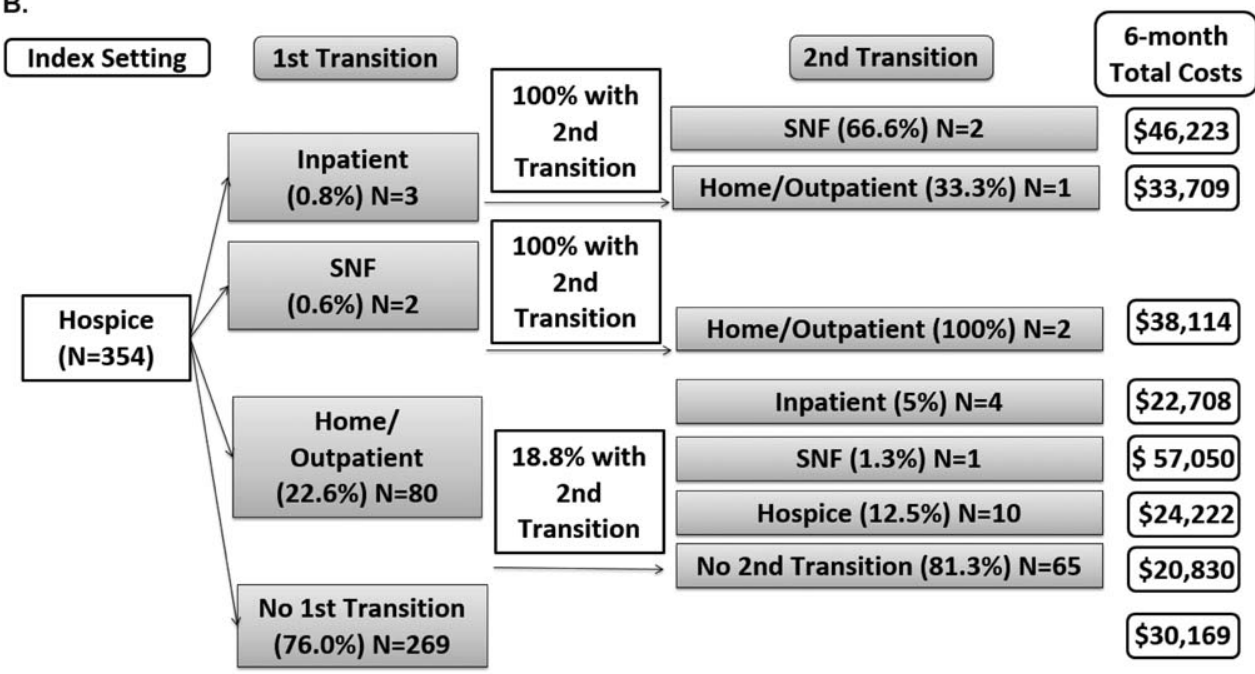

Figure 3. Transition from (A) skilled nursing facility, (B) hospice setting.

stay for the second time in the home or outpatient setting to the end of the 6 months after the AD diagnosis or next transition (if any) was 78 days, and participants incurred an average of $\$ 4,472(15.1 \%)$ of the average total cost during the 6-month postdiagnosis period (Supplementary Figure S1).

\section{Inpatient Setting $\rightarrow$ SNF Setting $\rightarrow$ Home or Outpatient Setting}

Average total healthcare expenditures were \$51,145 during the 6 months after $\mathrm{AD}$ diagnosis for participants in the second combination of transitions. These participants were hospitalized for an average of 8 days and incurred $24.8 \%(\$ 12,699)$ of the average total cost. Participants remained for an average of 43 days during their SNF stay, accounting for $39.9 \%(\$ 20,417)$ of the average total cost. The mean LOS for the home or outpatient setting to the end of the 6 months after AD diagnosis or next transition (if any) was 102 days, and participants incurred $9.7 \%$ $(\$ 4,985)$ of the average total cost (Supplementary Figure S2).
Home or Outpatient Setting $\rightarrow$ Inpatient Setting $\rightarrow$ SNF Setting

The average total Medicare expenditure of the third combination was \$52,183 over 6 months after AD diagnosis. The mean LOS in the index home or outpatient setting was 65 days, which accounted for $6.3 \%(\$ 3,296)$ of the average total cost before being admitted to the hospital. The average hospital stay was 8 days, which cost $25 \%$ $(\$ 13,263)$ of the average total cost. These individuals were later discharged to a SNF, where they remained for an average of 60 days, incurring an average of $32.9 \%$ $(\$ 17,185)$ of the average total cost in the 6 -month period (Supplementary Figure S3).

\section{Inpatient Setting $\rightarrow$ Home or Outpatient Setting $\rightarrow$ Inpatient Setting}

Individuals in the fourth combination incurred an average $\$ 50,780$ of costs during the 6 months after $\mathrm{AD}$ diagnosis. These individuals were hospitalized for an average of 8 days, resulting in an average cost of $\$ 11,283(22.2 \%$ of the average total cost). Participants remained for an 
average of 65 days in the home or outpatient setting before being re-admitted to the hospital, where they remained for an average of 6 days. The mean cost in the home or outpatient setting was $\$ 4,043$, which was approximately $8 \%$ of the average total cost; the average cost of the second inpatient setting was $\$ 11,124(21.1 \%$ of the average total cost) (Supplementary Figure S4).

\section{DISCUSSION}

A significant portion of individuals received their first $\mathrm{AD}$ diagnosis in a nonambulatory setting-particularly inpatient settings. Participants diagnosed in nonambulatory settings went through more transitions of care during the 6-month postdiagnosis period than those who were diagnosed in a home or outpatient settings. Overall economic burden was substantial for participants diagnosed in nonambulatory settings-an average of $\$ 41,468$ (results not shown) during the 6 months after AD diagnosis-whereas participants diagnosed in an ambulatory setting incurred an average of only $\$ 12,597$ during the same period.

The 30-day readmission rate after index hospitalization discharge was $22 \%$ in this analysis. In addition, the current study results indicate that, once an $\mathrm{AD}$ diagnosis is made, especially if a diagnosis is made in an inpatient setting, subsequent care is necessary. It is possible that these individuals were not adequately managed or were not given an adequate management postdischarge plan, which might have resulted in a high readmission rate. Furthermore, individuals with $\mathrm{AD}$ with other comorbidities need to be properly managed and treated for all of their conditions to prevent some of the extensive additional care. The cost of comorbid conditions in individuals with $\mathrm{AD}$ could explain some of the cost differences between index settings and transitions between settings. We did not evaluate the effect of the costs of comorbidities in the current analysis because it was beyond of the scope of the study.

Early detection of dementia is helpful in delivering appropriate care and offering direct benefits to individuals with dementia, ${ }^{11}$ and studies of hospital data have found that early detection of $\mathrm{AD}$ can be cost-saving. ${ }^{12-14}$ The observed benefit from the study findings suggests that participants managed in home and outpatient settings who did not have another transition during the 6 -month postdiagnosis period, were better managed and cost the healthcare system less. This highlights the need to reevaluate management of $\mathrm{AD}$ by preventing frequent hospitalizations with proactive outpatient care ${ }^{15}$ because the earlier an individual is managed, the longer he or she can avoid intensive healthcare resource encounters.

We also observed high cost burden for participants who transitioned to institutional care facilities, such as an inpatient setting or SNF, or those who transitioned twice to an inpatient setting. As reported above, $\mathrm{AD}$ was not listed as an admitting diagnosis for most of these individuals. Even when transitions from care settings where AD was initially diagnosed were assessed, $\mathrm{AD}$ was not observed to be the primary diagnosis for subsequent care settings. Furthermore, $\mathrm{AD}$ exacerbates other comorbid conditions, and individuals with $\mathrm{AD}$ tend not to adhere to management because of their cognitive impairment, which can complicate their care. ${ }^{16,17}$ Our study was exploratory and descriptive and provides a preliminary examination of how these comorbidities are present among $\mathrm{AD}$ patients across different healthcare settings. Dementia, even if it is not the primary reason for an episode of care, can have a profound effect on care delivery and outcomes ${ }^{18-20}$

The health status of an individual seeking care for an acute event such as hip fracture can be updated at the onset of care for the acute episode and during transitions to different care settings. A different approach might be developed for individuals seeking care for a chronic illness such as AD. Being a chronic disease, AD is difficult to study in terms of episodes of care because it is often difficult to define a start and end of an episode for a chronic disease. Even though our health economic approach to episodes might not easily transition into a bundled payment approach, they can help analyze transitions of care between healthcare settings for individuals with AD.

Although claims data are valuable for the effective assessment of real-world health information, there are inherent limitations in this study. The main challenge of existing bundles is their short duration because most cover services up to 90 days after hospital discharge for Medicare beneficiaries. ${ }^{21}$ Further studies should evaluate bundled payment using longer follow-up time frames if they are to be useful for chronic diseases. In most institutional settings, $\mathrm{AD}$ was not coded as an admitting diagnosis in the current analysis. Undercoding of $\mathrm{AD}$ in acute hospital and outpatient care settings could cause this, as previously discussed. ${ }^{22}$ Measures such as AD duration, disease severity and progression, stage of disease, cause of death, health behaviors, and caregiver information were lacking in the Medicare data, so study participants may represent individuals across the spectrum of dementia severity and may not reflect results for individuals with new onset of $\mathrm{AD}$. This study was descriptive, so factors such as age, sex, and comorbid conditions that could affect individuals' costs and transitions of care were not adjusted in a multivariate model. Methodologically, a multivariate analysis of a sequence of acute events as part of an overall episode is not straightforward because the pattern of such care will vary according to the individual. This study included fee-for-service beneficiaries enrolled in Medicare Parts A and B, which may limit generalizability of the study results to individuals enrolled in Medicare Part C. Studies are needed to determine the significance of the episode-of-care approach to analysis of the health economics and quality of care in Medicare Part $\mathrm{C}$ settings. At the time of this analysis, ICD-9 code 331.0 was a billable code. It is likely that the switch from ICD-9 to ICD-10 codes for disease classification will improve results and quality of care because ICD-10 codes are more specific to individual disease conditions, allowing for more effective disease management.

This analysis displays how an episode-of-care approach might be implemented using health economic data to identify individuals at risk of hospitalization and analyze care across the continuum of care for individuals with AD. This study highlights the number of transitions, time between each transition, and healthcare expenditures 
across different healthcare facilities for individuals newly diagnosed with $\mathrm{AD}$, which could help stakeholders and medical professionals to better manage individuals with $\mathrm{AD}$ in different healthcare settings and guide future research.

\section{ACKNOWLEDGMENTS}

Author Contributions: Fillit, Ambegaonkar, Yuce: data interpretation, manuscript preparation, critical revision of intellectual content. Fillit, Kariburyo, Black, Khandker, Xie: study concept design, data analysis and interpretation, manuscript preparation, critical revision of intellectual content. Baser: data acquisition and interpretation, critical revision of intellectual content.

Conflict of Interest: Christopher M. Black, Rezaul K. Khandker and Baishali M. Ambegaonkar were employees of Merck \& Co., Inc., Kenilworth, NJ, USA at the time of the analysis. Huseyin Yuce, Lin Xie, M. Furaha Kariburyo, and Onur Baser were employees of STATinMED Research, which is a paid consultant to Merck \& Co., Inc., Kenilworth, NJ, USA Dr. Howard Fillit was a consultant for Merck \& Co., Inc., Kenilworth, NJ, USA.

Sponsor's Role: This study was funded by Merck \& Co., Inc., Kenilworth, NJ, USA.

\section{REFERENCES}

1. Tsai TC, Greaves F, Zheng J, Orav EJ, Zinner MJ, Jha AK. Better patient care at high-quality hospitals may save medicare money and bolster episodebased payment models. Health Aff (Millwood) 2016;35:1681-1689.

2. Amico P, Pope GC, Meadow A, West P. Episode-based payment for the Medicare outpatient therapy benefit. Arch Phys Med Rehabil 2016;97: 1323-1328.

3. Mechanic RE. Opportunities and challenges for episode-based payment. New Engl J Med 2011;365:777-779.

4. Plassman BL, Langa KM, Fisher GG et al. Prevalence of dementia in the United States: The Aging, Demographics, and Memory Study. Neuroepidemiology 2007;29:125-32.

5. Alzheimer's Association 2013 Alzheimer's disease facts and figures. Alzheimers Dement 2013;9:208-245.

6. Alzheimer's Association 2014 Alzheimer's disease facts and figures. Alzheimers Dement 2014;10:e47-e92.

7. Alzheimer's Association 2015 Alzheimer's disease facts and figures. Alzheimers Dement 2015;11:332.

8. Alzheimer's Association 2016 Alzheimer's disease facts and figures. Alzheimers Dement 2016;12:459-509.

9. Housman TS, Williford PM, Feldman SR, et al. Nonmelanoma skin cancer: An episode of care management approach. Dermatolog Surg 2003;29: 700-711.

10. Lamberts H, Hofmans-Okkes I. Episode of care: A core concept in family practice. J Fam Pract 1996;42:161-168.

11. Bradford A, Kunik ME, Schulz P, Williams SP, Singh H. Missed and delayed diagnosis of dementia in primary care: Prevalence and contributing factors. Alzheimer Dis Assoc Disord 2009;23:306.
12. Bianchetti A, Scuratti A, Zanetti O et al. Predictors of mortality and institutionalization in Alzheimer disease patients 1 year after discharge from an Alzheimer dementia unit. Dementia 1995;6:108-112.

13. Zhao Y, Kuo TC, Weir $S$ et al. Healthcare costs and utilization for Medicare beneficiaries with Alzheimer's. BMC Health Serv Res $2008 ; 8: 1$.

14. Weimer DL, Sager MA. Early identification and treatment of Alzheimer's disease: Social and fiscal outcomes. Alzheimers Dement 2009;5:215-226

15. Lin PJ, Fillit HM, Cohen JT et al. Potentially avoidable hospitalizations among Medicare beneficiaries with Alzheimer's disease and related disorders. Alzheimers Dement 2013;9:30-38.

16. Torian L, Davidson E, Fulop G, Sell L, Fillit H. The effect of dementia on acute care in a geriatric medical unit. Int Psychogeriatr 1992;4:231-239.

17. Dillon C, Serrano CM, Castro D, Leguizamón PP, Heisecke SL, Taragano FE. Behavioral symptoms related to cognitive impairment. Neuropyschiatr Dis Treat 2013;9:1443-1455.

18. Chang A, Walter LC. Recognizing dementia as a terminal illness in nursing home residents: Comment on "Survival and comfort after treatment of pneumonia in advanced dementia." Arch Intern Med 2010;170: 1107-1109.

19. Killington M, Walker R, Crotty M. The chaotic journey: Recovering from hip fracture in a nursing home. Arch Gerontol Geriatr 2016;67:106-112.

20. Saver BG, Wang CY, Dobie SA, Green PK, Baldwin LM. The central role of comorbidity in predicting ambulatory care sensitive hospitalizations. Eur J Pub Health 2013;24:66-72.

21. Navathe AS, Song Z, Emanuel EJ. The next generation of episode-based payments. JAMA 2017;317:2371-2372.

22. Fillit H, Geldmacher DS, Welter RT, Maslow K, Fraser M. Optimizing coding and reimbursement to improve management of Alzheimer's disease and related dementias. J Am Geriatr Soc 2002;50:1871-1878.

\section{SUPPORTING INFORMATION}

Additional Supporting Information may be found in the online version of this article.

Table S1. Demographic and Clinical Characteristics of Newly Diagnosed AD patients (By Index Settings)

Table S2. Health Care Utilizations During the 6month Follow-up Period

Figure S1. Transition Between Home/Outpatient $\rightarrow$ Inpatient $\rightarrow$ Home/Outpatient

Figure S2. Transition Between Inpatient $\rightarrow$ SNF $\rightarrow$ Home/Outpatient

Figure S3. Transition Between Home/Outpatient $\rightarrow$ Inpatient $\rightarrow$ SNF

Figure S4. Transition Between Inpatient $\rightarrow$ Home/ Outpatient $\rightarrow$ Inpatient

Please note: Wiley-Blackwell is not responsible for the content, accuracy, errors, or functionality of any supporting materials supplied by the authors. Any queries (other than missing material) should be directed to the corresponding author for the article. 